\title{
Microstructure Patterns by Switching Spectroscopy Piezo-response Force Microscopy of Lead Free Perovskite-type Polycrystalline Thin Films
}

\author{
G. Herrera-Pérez ${ }^{1}$, O. Solis-Canto ${ }^{2}$, J. Holguin-Momaca ${ }^{3}$, S. Olive-Mendez ${ }^{3}$, E. Guerrero-Lestarjette ${ }^{2}$, \\ G. Tapia-Padilla ${ }^{1}$, A. Reyes-Rojas ${ }^{3}$, L. E. Fuentes-Cobas ${ }^{3}$ \\ 1. CONACYT Research Fellow. Centro de Investigación en Materiales Avanzados (CIMAV), Miguel de \\ Cervantes 120, Chihuahua, Chih., Mexico. \\ 2. Laboratorio Nacional de Nanotecnología (NanoTech). Centro de Investigación en Materiales \\ Avanzados (CIMAV), Miguel de Cervantes 120, Chihuahua, Chih., Mexico. \\ 3. Physics of Materials Department. Centro de Investigación en Materiales Avanzados (CIMAV), Miguel \\ de Cervantes 120, Chihuahua, Chih., Mexico.
}

The purpose of this work is to perform DC-based manipulations as a nanolithography tool to fabricate nanostructured patterns on lead free ferroelectric thin films (TF) with stoichiometry $\mathrm{Ba}_{1-\mathrm{x}} \mathrm{Ca}_{\mathrm{x}} \mathrm{Ti}_{0.9} \mathrm{Zr}_{0.1} \mathrm{O}_{3}$ (denoted in this work as BCZT), where $\mathrm{x}=0.1,0.15$ and 0.2 . To establish the evidence of ferroelectricity in BCZT, one has to probe the local domain configurations and demonstrate the switching of the polarization [1]. For this material, the switching corresponds positively and negatively to the polarized regions [2], and as an example, we use the CIMAV logo as template, Fig 1a.

Polarization switching in polycrystalline BCZT bulk and thin films on Pt-coated substrates was studied by switching spectroscopy piezo-response force microscopy (SS-PFM) in dual AC resonance tracking (DART) mode. Fig. 1b and 1c show the switching domains before and after of the hysteresis loop measurement in the region marked with a blue circle on the PFM amplitude micrographs for the BCZT$\mathrm{TF}$ with $\mathrm{x}=1$. In these micrographs, the white region was polarized downward, whereas, the orange dotted region was polarized upward, confirming the $180^{\circ}$ phase difference between the two domain configurations. The out-of-plane piezo-response was determined as a function of an applied voltage, and the PFM hysteresis loops, both in amplitude and phase are shown in Fig. 2a and $2 b$, respectively. Figure $2 \mathrm{a}$ exhibits a butterfly shape, whereas Fig. $2 \mathrm{~b}$ reveals a sharp $180^{\circ}$ inversion at the coercive voltages with a clear hysteresis. These results confirm the ferroelectric nature of BCZT-TF. The piezoelectric response $d_{33}$ was determined by analyzing the local amplitude measurements "off-state" through the following equation $\left(\mathrm{V}-\mathrm{V}_{1}\right) d_{33}=\mathrm{D}-\mathrm{D}_{1}$ [3]; shown in Fig. 2c. BCZT-TF shows a $d_{33}=2.2 \mathrm{pmV}^{-1}$ at the maximum voltage of $50 \mathrm{~V}$ and the coercive voltage was around $12.2 \mathrm{~V}$.

To complement the characterization of these electro-ceramics, Fig. 3a and $3 \mathrm{~b}$ show a comparison between the X-ray diffraction (XRD) for the target prepared modified Pechini method [4] and sintered at $1200{ }^{\circ} \mathrm{C}$ for $5 \mathrm{~h}$; and the grazing incidence XRD for BCZT-TF (obtained by radio frequency magnetron sputtering system), respectively. The Rietveld refinement for the XRD results suggest a perovskite-type structure with tetragonal phase ( $P 4 m m$ space group), that is compatible with the ferro (Fig. $3 \mathrm{c}$ ) - piezo electrical response. In summary, the polarization switching behavior in the ferroelectric BCZT-TF as a relevant tool to optimize the image of polarization reversal as a lithography technique to store nanostructured patterns was shown [5].

\section{References:}

[1] C Dubourdieu et al. Nat. Nanotechnol. 8 (2013), 748. 
[2] DB Li, D. Bonnell Annu. Rev. Mater. Res. 38 (2008), 351.

[3] P Bharathi, P Thomas, and KBR Varma J. Mater. Chem. C, 3 (2015), 4762.

[4] G Herrera-Pérez et al. J. Appl. Phys. 120 (2016), 094303.

[5] The authors acknowledge funding from the Basic Research Project CONACyT-SEP Grant No. 253605 and Cátedra CONACyT Grant No. 2563. Dr. Herrera would like to thank for SNI 1.
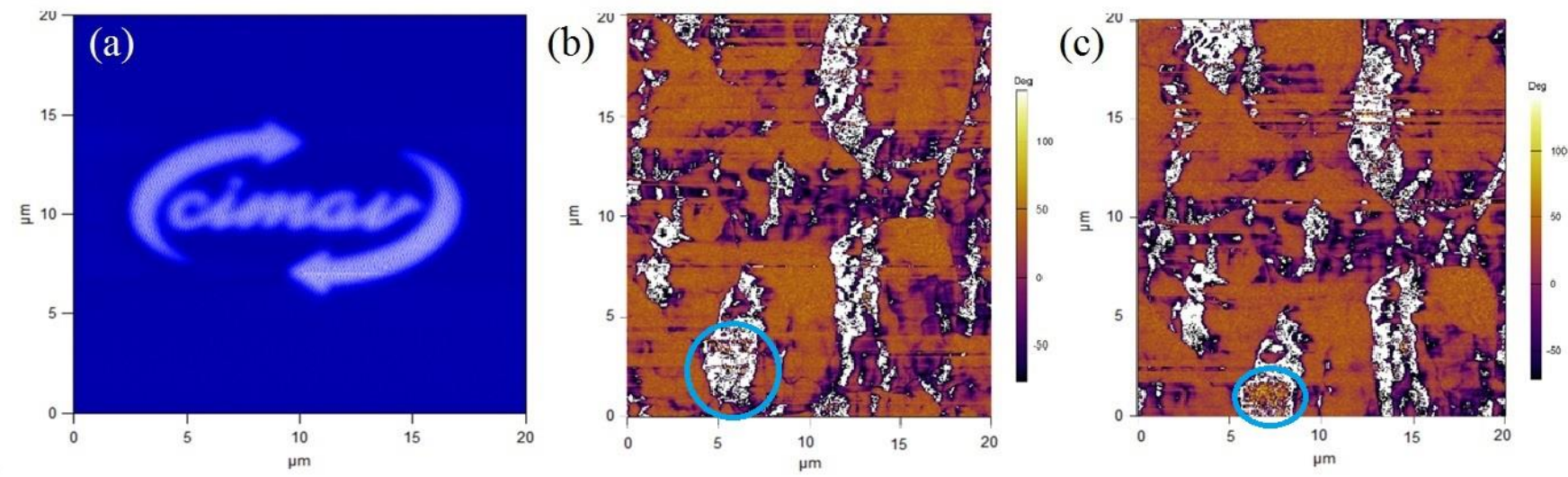

Figure 1. (a) PFM base lithography using CIMAV logo as template. Schematic of DART technique before (b) and after (c) make the hysteresis loop in the region marked with a blue circle.
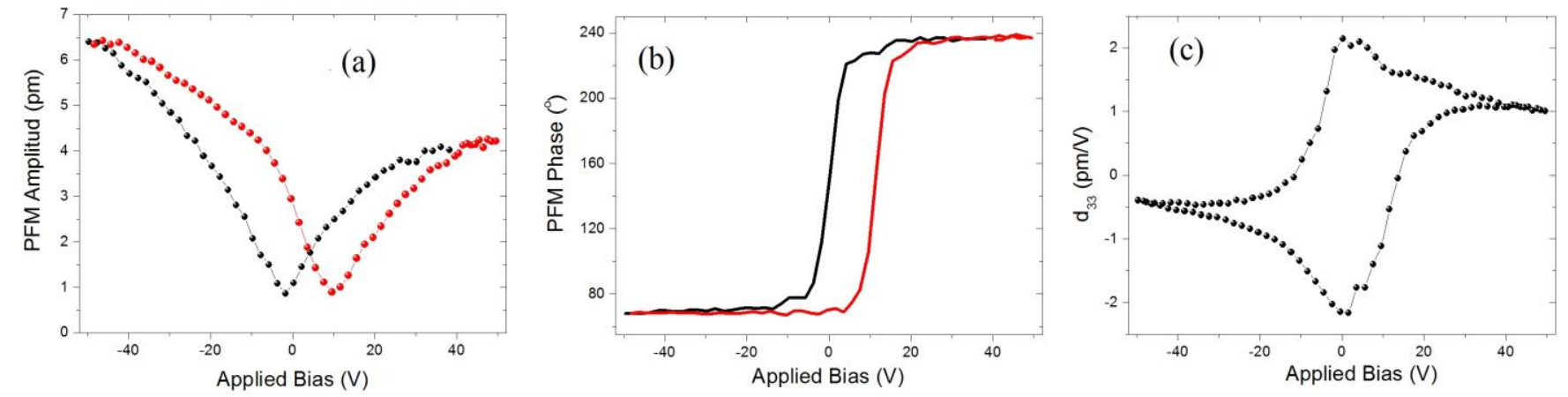

Figure 2. Local hysteresis loops measured by (a) PFM amplitude, (b) PFM phase, and (c) piezoresponse $\left(d_{33}\right)$ versus bias voltage for the BCZT-TF with $\mathrm{x}=1$.
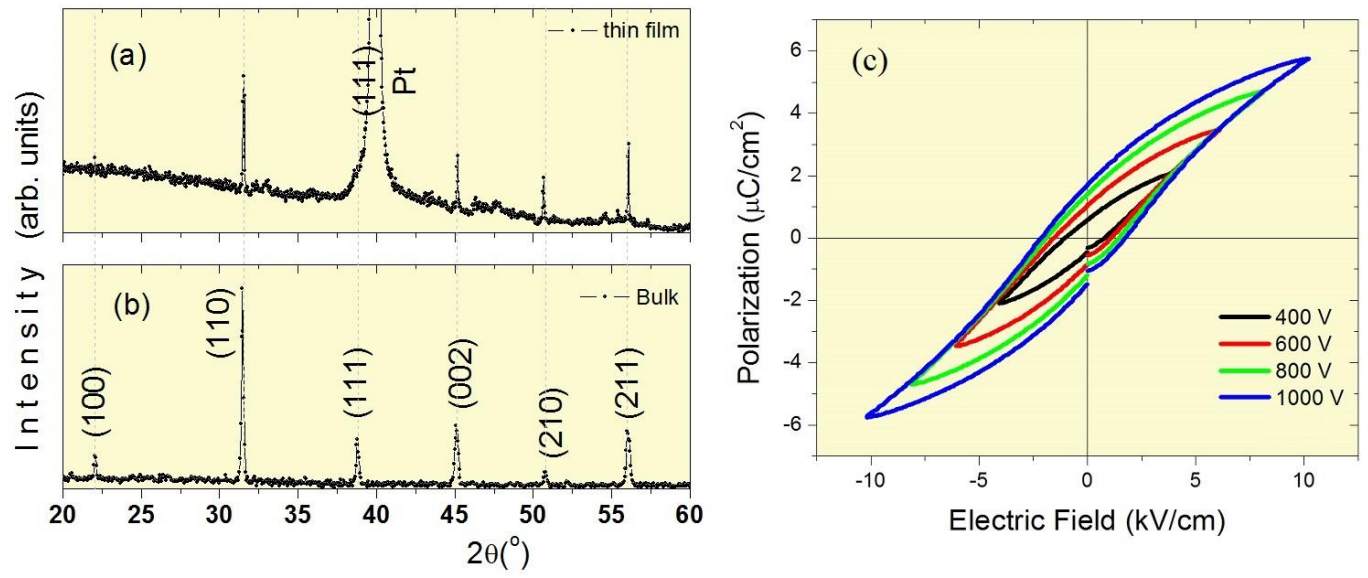

Figure 3. ( $\mathrm{a}$ and b) Comparison between XRD patterns for BCZT-TF and bulk, respectively. (c) Macroscopic polarization-electric field hysteresis loops at different voltages for the BCZT target. 